\title{
Supersymmetry at the LHC: Searches, Discovery Windows, and Expected Signatures
}

Darin Acosta, University of Florida

(on behalf of the ATLAS and CMS Collaborations)

\section{Introduction}

Supersymmetry (SUSY) postulates a symmetry between fermions and bosons, which is a necessary ingredient to String Theories, solves the hierarchy problem in the Standard Model, and provides for Grand Unification of the coupling constants. It postulates scalar counterparts to the known fermions - socalled sleptons $(\tilde{\ell})$ and squarks $(\tilde{q})$ - and fermion counterparts to the gauge bosons - so-called neutralinos $\left(\tilde{\chi}_{1,2,3,4}^{0}\right)$, charginos $\left(\tilde{\chi}_{1,2}^{ \pm}\right)$, and gluinos $(\tilde{g})$. SUSY must be broken since no direct evidence exists at low mass, but the SUSY particles should have a mass less than a few $\mathrm{TeV} / \mathrm{c}^{2}$ to avoid finetuning problems. In the Minimal Supersymmetric Model (MSSM), one usually supposes that $R$-parity, defined as +1 for particles and -1 for sparticles, is conserved, which leads to the lightest supersymmetric particle (LSP) being stable. MSSM introduces 105 new parameters, so it is conventional to consider more constrained models in order to express the limits and sensitivities of experiments.

In the Minimal Supergravity model (mSUGRA), universal gravitational interactions break SUSY. At the Grand Unification scale $\left(\sim 10^{16} \mathrm{GeV}\right)$, the gauginos and scalars have common masses and couplings. The five free parameters are the common gaugino mass $\left(m_{1 / 2}\right)$, the common scalar mass $\left(m_{0}\right)$, the common scalar trilinear coupling $\left(A_{0}\right)$, the ratio of the vacuum expectation values of the Higgs doublets $(\tan \beta)$, and the sign of the Higgsino mixing parameter $(\mu)$. Typically, the squarks and gluinos have masses larger than the charginos and neutralinos. Also, $m\left(\tilde{\chi}_{1}^{ \pm}\right) \approx m\left(\tilde{\chi}_{2}^{0}\right) \approx 2 m\left(\tilde{\chi}_{1}^{0}\right)$.

In Gauge Mediated Symmetry Breaking (GMSB), Standard Model gauge interactions break SUSY at a scale $\sqrt{F}$ much less than in mSUGRA. Particles acquire a mass from gauge interactions at a messenger scale $M_{\mathrm{m}} \sim 1000 \mathrm{TeV}$. The free parameters of the model include the number of $S U(5)$ messenger fields, $n$, and $\Lambda=F / M_{\mathrm{m}}$. In GMSB the gravitino is the LSP, and the phenomenology depends on whether the next-to-lightest supersymmetric particle (NLSP) is a neutralino $(n=1$, low $\tan \beta)$ or a slepton $(n>1$, high $\tan \beta)$ and whether or not the NLSP decays promptly inside the detector.

The Large Hadron Collider (LHC), under construction at CERN, will be the first collider to cross a new energy threshold $(\sqrt{s}=14 \mathrm{TeV})$ in almost two decades when it begins operation in 2007. During the first two years of 
operation, the instantaneous luminosity is expected to be $2 \times 10^{33} \mathrm{~cm}^{-2} \mathrm{~s}^{-1}$ $\left(\approx 20 \mathrm{fb}^{-1} /\right.$ year $)$, and ultimately it is expected to reach $10^{34} \mathrm{~cm}^{-2} \mathrm{~s}^{-1}(\approx$ $100 \mathrm{fb}^{-1} /$ year $)$.

\section{Trigger Strategies}

The beam crossing frequency of the LHC will be $40 \mathrm{MHz}$. At high luminosity, approximately 20 inelastic proton collisions will occur every beam crossing, yielding a collision rate of nearly $1 \mathrm{GHz}$. This must be reduced to about $100 \mathrm{~Hz}$ by the experiments for data acquisition, which makes triggering at the LHC a challenge. The CMS Collaboration has recently performed detailed detector simulations to understand the performance of its high-level triggers [1]. The rates, thresholds, and efficiencies for some representative SUSY searches are discussed here.

Figure 1a shows the simulated single-jet trigger rate for a simple cone algorithm $(R=0.5)$ as a function of the jet $E_{\mathrm{T}}$ for both low and high luminosity. The calorimeter-based trigger is assumed to have been calibrated to particle-level jets. To reduce the inclusive single-jet rate to $\approx 1 \mathrm{~Hz}$ at low luminosity requires an $E_{\mathrm{T}}$ threshold of approximately $0.5 \mathrm{TeV}$. In addition, Fig. $1 \mathrm{~b}$ shows the $E_{\mathrm{T}}^{\mathrm{miss}}$ trigger rate as a function of the applied threshold for both low an high luminosity. At the trigger level, the $E_{\mathrm{T}}^{\text {miss }}$ rate is dominated by detector resolution, and is thus higher than the generated $E_{\mathrm{T}}^{\text {miss }}$ rate. $\mathrm{A}$ threshold of approximately $125 \mathrm{GeV}$ is required to reduce the rate to $\approx 1 \mathrm{~Hz}$ at low luminosity.

With such high thresholds, it is not known a priori whether one can efficiently trigger on SUSY events at the LHC, especially for low masses and for models where $R$-parity is not conserved. To address this issue, the CMS Collaboration studied several prototype trigger menus and checked their performance against several possible SUSY scenarios. The points in mSUGRA space considered at low luminosity are near the expected Tevatron II reach:

1. $m_{0}=20 \mathrm{GeV}$ and $m_{1 / 2}=190 \mathrm{GeV}$, yielding $\sigma=181 \mathrm{pb}$

2. $m_{0}=150 \mathrm{GeV}$ and $m_{1 / 2}=180 \mathrm{GeV}$, yielding $\sigma=213 \mathrm{pb}$

3. $m_{0}=300 \mathrm{GeV}$ and $m_{1 / 2}=150 \mathrm{GeV}$, yielding $\sigma=500 \mathrm{pb}$

It was assumed that $\tan \beta=10, A_{0}=0$ and $\mu>0$ in all cases, and both $R_{\mathrm{p}}$ conservation and non-conservation were studied. In the case of $R_{\mathrm{p}}$ non-conservation, the most difficult experimental case was considered: $\tilde{\chi}_{1}^{0} \rightarrow 3$ jets.

An example of a simple trigger menu that can be applied at low luminosity in the second-level trigger of CMS is a 1-jet plus $E_{\mathrm{T}}^{\text {miss }}$ trigger with thresholds at 160 and $90 \mathrm{GeV}$, respectively, and a 4-jet trigger with a threshold of $90 \mathrm{GeV}$ applied to each jet. The requirements applied in the first-level electronic trigger are somewhat more relaxed than these. This yields a background trigger rate of $11 \mathrm{~Hz}$ at low luminosity according to detailed detector 
simulations. The efficiencies for the mSUGRA points with $R_{\mathrm{p}}$ conserved are $69 \%, 68 \%$, and $44 \%$ for the first, second, and third points, respectively; whilst the corresponding efficiencies with $R_{\mathrm{p}}$ violated are $46 \%, 41 \%$, and $26 \%$. In the case of $R_{\mathrm{p}}$ conservation, the efficiency can be further improved with various cuts on topological and global variables. At high luminosity, one expects to explore larger sparticle masses at larger $m_{0}$ and $m_{1 / 2}$, and triggering becomes more efficient despite higher thresholds.
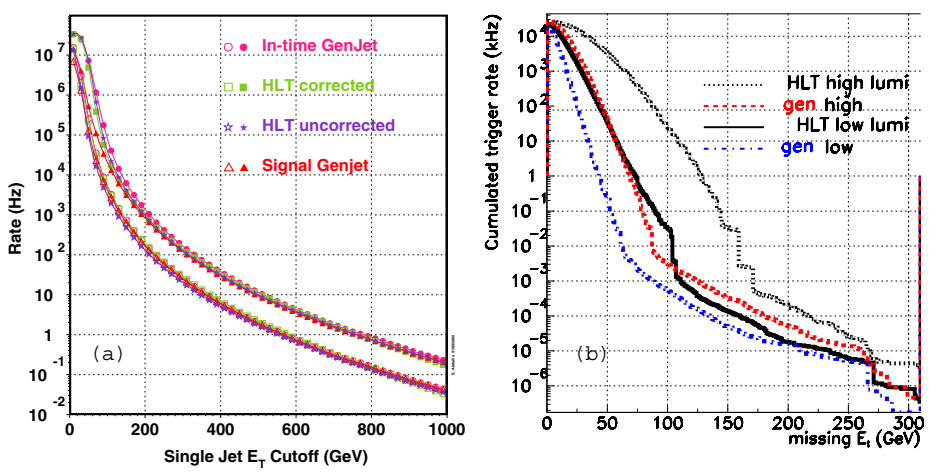

Fig. 1. (a) Simulated rates for a single jet trigger at low luminosity (open symbols, lower curve) and high luminosity (closed symbols, upper curve). (b) Simulated rates for a $E_{\mathrm{T}}^{\text {miss }}$ High-Level Trigger compared to the generator-level rates at both low and high luminosity.

\section{3 mSUGRA}

\subsection{Inclusive Searches}

Both ATLAS and CMS have performed studies [2][3] to determine the reach in mSUGRA parameter space for an inclusive search for SUSY particle production and decay. These studies use fast, parameterized simulations of the detector performance because of the large number of events to be simulated $\left(\approx 10^{8}\right)$. The studies are classified according to the final state being sought:

- $E_{\mathrm{T}}^{\mathrm{miss}}$ : inclusive jets and missing transverse energy without any cuts regarding the presence of leptons

- $0 \ell$ : the same, but leptons are vetoed

- $1 \ell: 1$ lepton required

- $2 \ell \mathrm{OS}: 2$ leptons with opposite sign required

- $2 \ell \mathrm{SS}: 2$ leptons with the same sign required

- $3 \ell: 3$ leptons required 
For each final state, cuts are varied in several categories to optimize $S / \sqrt{S+B}$ in a grid of mSUGRA points, and a map is determined for where this quantity exceeds 5 for a given integrated luminosity. No attempt is made to reconstruct the SUSY particle decays contributing to the excess.

In the CMS study, for example, the cuts varied include the number of jets, $E_{\mathrm{T}}^{\text {miss }}$, jet $E_{\mathrm{T}}, \Delta \phi\left(\ell, E_{\mathrm{T}}^{\mathrm{miss}}\right)$, circularity, and muon isolation. Cuts common to all mSUGRA grid points in the CMS study are $E_{\mathrm{T}}^{\text {miss }}>200 \mathrm{GeV}$ and at least 2 jets with $E_{\mathrm{T}}>40 \mathrm{GeV}$ and $|\eta|<3$. Leptons must have $|\eta|<2.4$ and $E_{\mathrm{T}}>20 \mathrm{GeV}(10 \mathrm{GeV})$ for electrons (muons). Electrons must be isolated. The ATLAS study applies a similar search strategy.

Figure 2 shows the discovery reach in the $\left(m_{0}, m_{1 / 2}\right)$ plane for several choices of the other mSUGRA parameters and for several choices of the integrated luminosity. The greatest sensitivity at high $\tan \beta$ in the CMS study comes from the inclusive jets and $E_{\mathrm{T}}^{\mathrm{miss}}$ search. Squarks and gluinos are probed to a mass of $2.5 \mathrm{TeV} / c^{2}$ in one year of design luminosity $\left(100 \mathrm{fb}^{-1}\right)$, and reaching $3 \mathrm{TeV} / c^{2}$ with more than $300 \mathrm{fb}^{-1}$. However, with just $1 \mathrm{fb}^{-1}$ collected in the first couple of months of LHC operation, masses already can be probed up to $1.5 \mathrm{TeV} / c^{2}$.
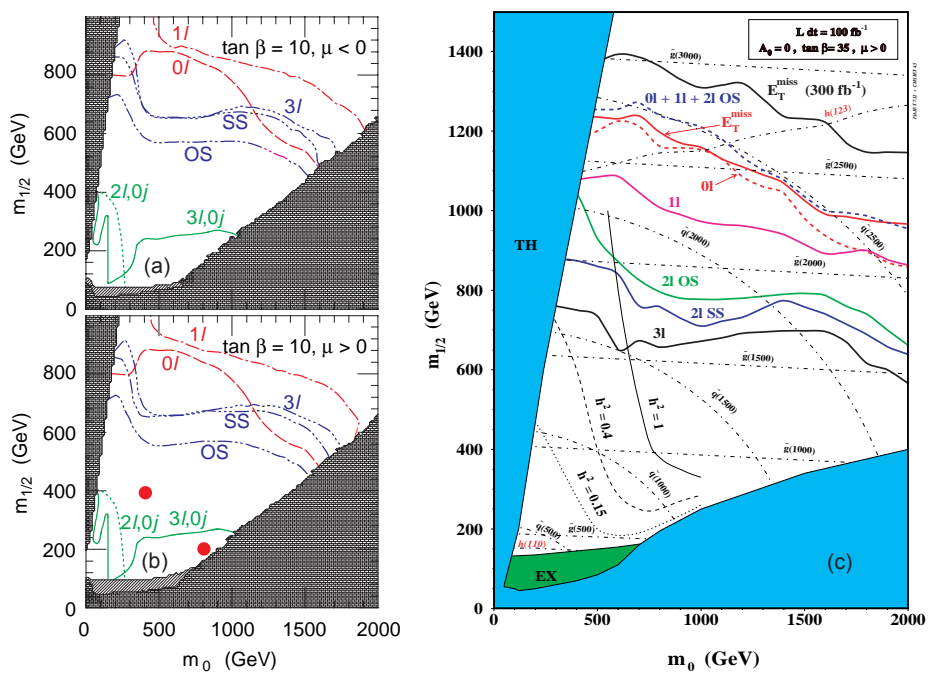

Fig. 2. (a) SUSY discovery reach in the $\left(m_{0}, m_{1 / 2}\right)$ plane, expressed as a $5 \sigma$ excess, for several final state analyses from the ATLAS study assuming $10 \mathrm{fb}^{-1}$ of collected data. The remaining mSUGRA parameters are $\tan \beta=10, A_{0}=0$ and $\mu>0$. (b) The same for $\mu<0$. (c) The same from the CMS study for $100 \mathrm{fb}^{-1}$ of data and for mSUGRA parameters $\tan \beta=35, A_{0}=0$ and $\mu>0$. Curves of constant $\tilde{q}$ and $\tilde{g}$ mass are also shown. 


\subsection{Exclusive Sparticle Reconstruction}

In contrast to the inclusive SUSY searches described above, partial or full reconstruction of SUSY particle decays is also possible at the LHC. For example, one can measure the invariant mass distribution of opposite sign, same flavor leptons as evidence for the decay $\tilde{\chi}_{2}^{0} \rightarrow \tilde{\chi}_{1}^{0} \ell^{+} \ell^{-}$or $\tilde{\chi}_{2}^{0} \rightarrow \tilde{\ell}^{+} \ell^{-} \rightarrow$ $\tilde{\chi}_{1}^{0} \ell^{+} \ell^{-}$. The $\tilde{\chi}_{2}^{0}$ typically would be most prevalent in $\tilde{q}$ and $\tilde{g}$ cascade decays at the LHC. Although the mass of the $\tilde{\chi}_{2}^{0}$ cannot be completely reconstructed because the $\tilde{\chi}_{1}^{0}$ is undetected, the endpoint of the mass spectrum exhibits a sharp edge. The endpoint of the 3-body decay is simply $m_{\ell \ell}^{\max }=m\left(\tilde{\chi}_{2}^{0}\right)-m\left(\tilde{\chi}_{1}^{0}\right)$, whereas the endpoint of the 2-body decay is given by $m_{\ell \ell}^{\max }=\left[m^{2}\left(\tilde{\chi}_{2}^{0}\right)-m^{2}(\tilde{\ell})\right]^{1 / 2}\left[m^{2}(\tilde{\ell})-m^{2}\left(\tilde{\chi}_{1}^{0}\right)\right]^{1 / 2} / m(\tilde{\ell})$.

Figure 3 illustrates the shape of the di-lepton mass spectrum, and its significance over the Standard Model background in $30 \mathrm{fb}^{-1}$, in an ATLAS study [2] of the mSUGRA point: $m_{0}=800 \mathrm{GeV} / c^{2}, m_{1 / 2}=200 \mathrm{GeV} / c^{2}$, $\tan \beta=10, A_{0}=0$ and $\mu>0$. In this analysis, two opposite sign electrons or muons with $|\eta|<2.5$ and $p_{\mathrm{T}}^{\ell 1, \ell 2}>(20,10) \mathrm{GeV} / c$ are required in addition to $E_{\mathrm{T}}^{\mathrm{miss}}>200 \mathrm{GeV}$ and four jets with $p_{\mathrm{T}}^{j 1}>100 \mathrm{GeV} / c$ and $p_{\mathrm{T}}^{j 2, j 3, j 4}>50 \mathrm{GeV} / c$. The reconstructed endpoint agrees with the generated mass difference of $68 \mathrm{GeV} / c^{2}$. A $Z^{0}$ peak is also visible from SUSY cascade decays.

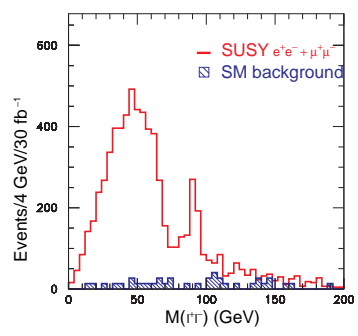

Fig. 3. Expected di-lepton mass spectrum in a search for $\tilde{\chi}_{2}^{0} \rightarrow \tilde{\chi}_{1}^{0} \ell^{+} \ell^{-}$in the ATLAS study.

The di-lepton reconstruction also forms a basis for reconstructing the decay of the scalar bottom quark (sbottom), which in turn may arise from the decay of a gluino: $\tilde{g} \rightarrow \tilde{b} b \rightarrow \tilde{\chi}_{2}^{0} b b$. A recent CMS study has explored the possibility of reconstructing the mass distribution of $\tilde{b}$ and $\tilde{g}$ decays for one of the proposed [4] mSUGRA benchmark points: $m_{0}=100 \mathrm{GeV} / c^{2}$, $m_{1 / 2}=250 \mathrm{GeV} / c^{2}, \tan \beta=10, A_{0}=0$ and $\mu>0$.

Reconstruction of the SUSY decay chain in the CMS study starts with selecting di-lepton events within a $16 \mathrm{GeV} / c^{2}$ window around the di-lepton endpoint, such that the $\tilde{\chi}_{1}^{0}$ is essentially at rest in the $\tilde{\chi}_{2}^{0}$ rest frame. The momentum of the $\tilde{\chi}_{2}^{0}$ is thus related to the momentum of the lepton pair by 
the factor $\left[1+m\left(\tilde{\chi}_{1}^{0}\right) / m\left(\ell^{+} \ell^{-}\right)\right]$. The $\tilde{\chi}_{1}^{0}$ mass can be approximated by the $\ell^{+} \ell^{-}$endpoint, without too much impact on the $\tilde{b}$ reconstruction.

To reconstruct $\tilde{b}$, the most energetic $b$-jet with $E>250 \mathrm{GeV}$ and $|\eta|<2.4$ is added to the lepton pair. The $b$-jet is identified by $\geq 2$ tracks with an impact parameter significance greater than $3 \sigma$. Figure 4 a shows the distribution of the reconstructed $\tilde{b}$ mass in $10 \mathrm{fb}^{-1}$ of data after the additional cuts $E_{\mathrm{T}}^{\text {miss }}>$ $150 \mathrm{GeV}$ and $E_{\ell \ell}>100 \mathrm{GeV}$, which reduce the Standard Model background and suppress leptons from SUSY decays other than from $\tilde{\chi}_{2}^{0}$, respectively. The fitted mass of $480 \mathrm{GeV} / c^{2}$ is close to the two eigenstate masses of 496 and $524 \mathrm{GeV} / c^{2}$. The $\tilde{g}$ is reconstructed by associating a second $b$-jet closest in $\phi$ to the reconstructed $\tilde{b}$. Figure $4 \mathrm{~b}$ shows the reconstructed $\tilde{b}$ mass, where the fitted mass of $585 \mathrm{GeV} / c^{2}$ is close to the generated value of $595 \mathrm{GeV} / c^{2}$.
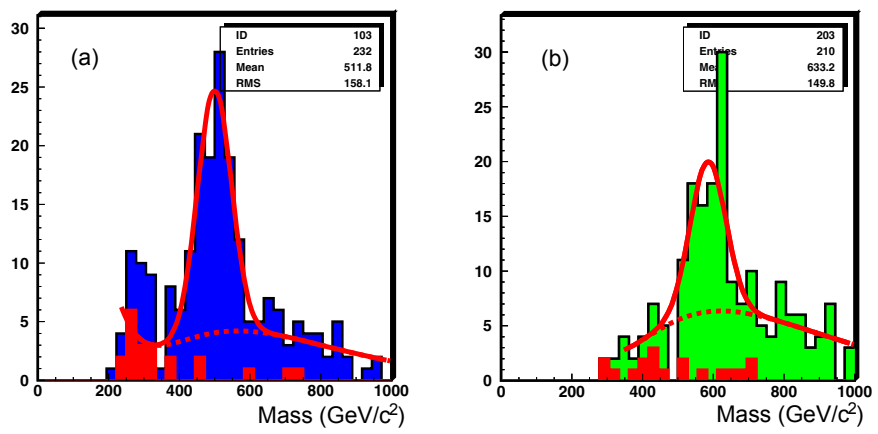

Fig. 4. Reconstructed distributions for (a) $M\left(\ell^{+} \ell^{-} b\right)$ and (b) $M\left(\ell^{+} \ell^{-} b b\right)$ expected in a sbottom and gluino search from the CMS study assuming $10 \mathrm{fb}^{-1}$ (see text for details).

\section{GMSB}

In GMSB the slepton could be the NLSP and quasi-stable with $c \tau$ larger than the detector dimensions. Identification of such heavy leptons can be achieved by using the drift-tube muon systems of ATLAS and CMS, instrumented with TDCs for drift-time measurements, essentially as time-of-flight detectors with a time resolution of order $1 \mathrm{~ns}$. Figure 5 a shows the results of a simulation by CMS on the measurement of $1 / \beta$ versus the momentum $p$ for several slepton masses, and Fig. 5b shows the corresponding reconstructed mass distributions from the measurement of $1 / \beta$ and $p$. The simulation assumes $n=3$ messenger fields, $\tan \beta=45, M_{\mathrm{m}} / \Lambda=200$, and $\Lambda=50-300 \mathrm{TeV}$. The analysis study requires two "muons" reconstructed by the barrel muon system $(|\eta|<1)$ with $p_{\mathrm{T}}>45 \mathrm{GeV} / c$ and $M_{\ell \ell}>97 \mathrm{GeV} / c^{2}$. The shown mass distributions correspond to the number of detected events expected with an integrated 
luminosity of 1,10 , and $100 \mathrm{fb}^{-1}$ for the three simulated slepton masses of 114,303 , and $636 \mathrm{GeV} / c^{2}$, respectively. For one year of design luminosity at the LHC, this study shows a sensitivity to slepton masses from 90 to $700 \mathrm{GeV} / c^{2}$.

If the neutralino is the NLSP in GMSB, then it will decay radiatively to a gravitino and photon. A finite lifetime will lead to an electromagnetic shower that does not point to the nominal collision vertex. The fine angular resolution of the ATLAS liquid argon calorimeter provides excellent vertex resolution of the order of $1 \mathrm{~cm}$, based on a study of $H^{0} \rightarrow \gamma \gamma$. In a GMSB study, the nonobservation of any non-projective photons excludes neutralino decays with $c \tau<100 \mathrm{~km}$ in $30 \mathrm{fb}^{-1}$ for $n=1, M_{\mathrm{m}}=500 \mathrm{TeV}$, and $\Lambda=90 \mathrm{TeV}$.
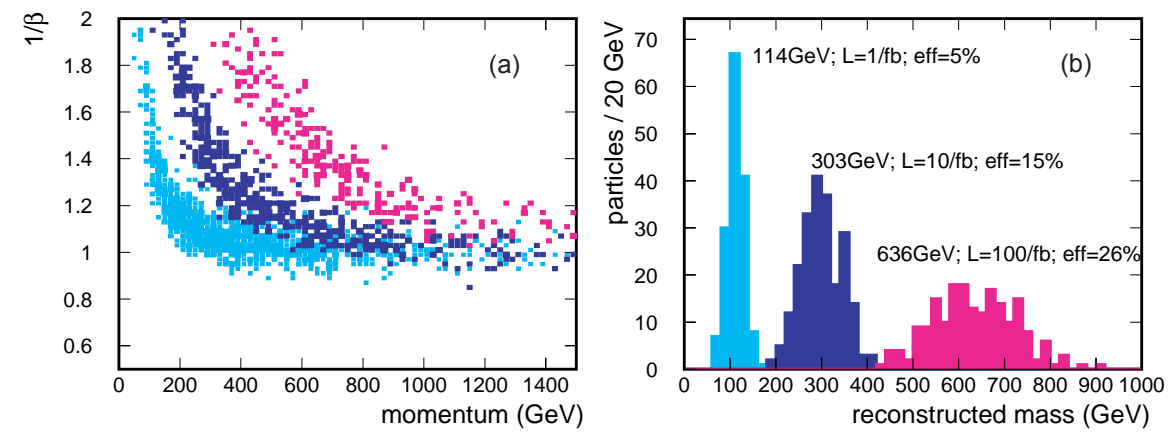

Fig. 5. (a) Simulation of $1 / \beta$ vs. $p$ for heavy leptons using the CMS drift-tube muon system. (b) Corresponding reconstructed mass distributions.

\section{Acknowledgements}

I am grateful to S.Abdullin, M.Chiorboli, D.Denegri, F.Gianotti and G.Wrochna for their assistance.

\section{References}

1. CMS Collaboration, "The Trigger and Data Acquisition Project, Technical Design Report, Volume 2, Data Acquisition and High Level Triggers," in preparation.

2. ATLAS Collaboration, "Detector and Physics Performance Technical Design Report," CERN/LHCC 99-15.

3. M. Dzelalija, Z. Antunovic, S. Abdullin and F. Charles, Mod. Phys. Lett. A15 (2000) 465; S. Abdullin and F.Charles, Nucl. Phys. B547 (1999) 60; S.Abdullin et al., "Discovery Potential for Supersymmetry in CMS," CMS Note 1998/006.

4. M. Battaglia et al., Eur. Phys. J. C22 (2001) 535. 